junction (Resnick and Niwayama, 1978). Such trauma may predispose to intravertebral herniation of portions of the disc producing cartilaginous or Schmorl's nodes. In rheumatoid arthritis, apophyseal joint instability and vertebral body osteoporosis may contribute to the incidence of such cartilaginous node formation. The similarity of radiographically evident rheumatoid discovertebral alterations (such as disc space loss, subchondral radiolucent vertebral lesions, and reactive bone sclerosis), to changes accompanying other disorders characterised by cartilaginous node formation is noteworthy. These latter disorders include any process which weakens either the cartilaginous end-plate of the vertebral body or the subchondral bone such as trauma, degenerative disease of the nucleus pulposus (intervertebral osteochondrosis) (Fig. 1), metabolic and endocrine disorders (Resnick and Niwayama, 1976). Furthermore, direct inspection of thoracic and lumbar spine specimens in some rheumatoid cadavers has documented the presence of cartilaginous nodes within sclerotic vertebral bodies, about narrowed intervertebral discs (Fig. 2). The resulting radiological picture in these cadavers simulates infection, although the vertebral outline is generally better defined than in patients with infective spondylitis.
In any case, the observation that thoracolumbar spine changes occur in rheumatic arthritis is certainly important and should stimulate further investigation in order to outline the pathogenesis of these lesions, investigation that requires close radiological-pathological correlation

DONALD RESNICK Department of Radiology, Veterans Administration Hospital, 3350 La Jolla Village Drive, San Diego, California 92161, USA

\section{References}

Martel, W. (1977). Pathogenesis of cervical discovertebral destruction in rheumatoid arthritis. Arthritis and Rheumatism, 20, 1217-1225.

Resnick, D., and Niwayama, G. (1976). Subchondral resorption of bone in renal osteodystrophy. Radiology, 118, 315-321.

Resnick, D., and Niwayama, G. (1978). Intravertebral disc herniations: cartilaginous (Schmorl's) nodes. Radiology, 126, 57-65.

Sims-Williams, H., Jayson, M. I. V., and Baddeley, H. (1977). Rheumatoid involvement of the lumbar spine. Annals of the Rheumatic Diseases, 36, 524-531.

\title{
Serum copper levels in rheumatoid arthritis
}

Sir,

Recent correspondence concerning the level and distribution of copper in the serum of patients undergoing therapy for rheumatoid arthritis (Bajpayee, 1975; Sorensen, 1976a) and observations that copper complexes of anti-inflammatory drugs are more active in animal models than the drugs themselves (Sorensen, 1976b) suggest that copper may possibly play an important, if little understood, role in the inflammatory process. Accordingly, the serum copper levels of a group of patients with rheumatoid arthritis who were undergoing therapy with different drug regimes were measured (Table). All subjects in the group had been treated with the drug indicated in the Table for at least 1 month before analysis of a sample of serum for copper by atomic absorption spectrometry using carbon furnace atomisation (Kamel et al., 1978). The results suggest that there is a correlation between serum copper level and drug therapy and, in particular, the levels found with aspirin and indomethacin are significantly different from non-rheumatoid controls at the $0.1 \%$ level.
We attempted to assess the effect of the duration of therapy over a period of 6 months by following the serum copper levels of selected patients who remained for that length of time on each drug listed except gold. Penicillamine produced a definite reduction in the average serum value during this period and, indeed, in some patients a level close to that of the controls was achieved. With aspirin, indomethacin, and levamisole there were some variations but no obvious trends were observed with these drugs in the time scale of this investigation. Previous studies have referred to groups of 'untreated' rheumatoids (Bajpayee, 1975; Sorensen, 1976a) but in our case all patients had been treated in some manner previously. Their copper levels before hospital treatment varied from $2 \cdot 15$ to $1 \cdot 11 \mathrm{mg} / \mathrm{ml}$.

The correlation between drug therapy and copper level was somewhat unexpected. Serum copper variations have been noted for quite a wide range of physiological conditions such as sex, pregnancy and contraception, stress and diurnal rhythms and these effects might have been expected to obscure any drug-related changes. However,

Table Serum copper levels of patients with rheumatoid arthritis undergoing therapy with different drugs

\begin{tabular}{lllllll}
\hline & Controls & Indomethacin & Aspirin & Levamisole & Penicillamine & Gold \\
\hline Number & 17 & 21 & 15 & 49 & 7 \\
Mean copper level $(\mu \mathrm{g} / \mathrm{ml})$ & 1.03 & 1.65 & 1.61 & 1.37 & 1.32 & 1.65 \\
Standard deviation & 0.15 & 0.20 & 0.18 & 0.28 & 0.18 & 0.42 \\
\hline
\end{tabular}


no pregnant women and none on oral contraceptives were included in this study and the drug-related differences found are quite large. (For example, differences of 0.16 and $0.19 \mathrm{Cu} \mu \mathrm{g} / \mathrm{ml}$ between male and female groups were reported in recent studies [Barnet and Brozovic, 1975, Sorensen 1974).

Therefore, serum copper levels, which are known to vary quite widely in patients with rheumatoid arthritis, appear to be stabilised by conventional drug therapies into certain bands which, with the possible exception of penicillamine, are higher than that of the controls. Further, the second-line drugs, penicillamine and levamisole, produce significantly lower copper levels than more standard anti-inflammatories such as aspirin and indomethacin.

D. H. Brown, A. El Ghobarey, W. E. SMITH, and J. TEAPE Department of Pure and Applied Chemistry, University of Strathclyde, and Centre for Rheumatic Diseases, Baird Street, Glasgow

\section{References}

Bajpayee, D. P. (1975). Significance of plasma copper a ceruloplasmin concentration in rheumatoid arthris. Annals of the Rheumatic Diseases, 34, 162.

Barnet, M. D., and Brozovic, B. (1975). A simple aut:mated micro-method for measuring serum copper. Cliniegl Chimica Acta, 58, 295-298.

Kamel, H., Teape, J., Brown, D. H., Ottaway, J. M., a⿳亠口冋 Smith, W. E. (1978). The determination of copper $\overline{\overline{c o n}}$ ultrafiltrate by atomic absorption spectrometry usi carbon furnace atomisation. Analyst. (In press.)

Sorensen, J. R. J. (1974). Trace substances in environmenf health. In VIII Symposium, ed. by D. D. Hamphill, p. 305. University of Missouri, Columbia.

Sorensen, J. R. J. (1976a). Correspondence. Annals of the Rheumatic Diseases, 35, 186.

Sorensen, J. R. J. (1976b). Copper chelates as possiaje active forms of the anti-arthritic agents. Journal of Medi $\mathbf{a} l$ Chemistry, 19, 135.

Zackheim, H. S., and Wolf, P. (1972). Serum copper in psoriasis and other dermatosis. Journal of Investigat $\overrightarrow{k f}$ Dermatology, 58, 28-32. 